Bull. Korean Math. Soc. 49 (2012), No. 1, pp. 89-98

http://dx.doi.org/10.4134/BKMS.2012.49.1.089

\title{
CHARACTERIZATIONS OF BESOV SPACES \\ IN THE UNIT BALL
}

\author{
SONGXIAO LI
}

ABSTRACT. In this paper we obtain some new characterizations of Besov spaces on the unit ball of $\mathbb{C}^{n}$. These characterizations are also completely new even in the settings of the unit disk.

\section{Introduction}

Let $B$ be the unit ball of $\mathbb{C}^{n}$. Let $z=\left(z_{1}, \ldots, z_{n}\right)$ and $w=\left(w_{1}, \ldots, w_{n}\right)$ be points in $\mathbb{C}^{n}$, we write

$$
\langle z, w\rangle=z_{1} \bar{w}_{1}+\cdots+z_{n} \bar{w}_{n},|z|=\sqrt{\left|z_{1}\right|^{2}+\cdots+\left|z_{n}\right|^{2}} .
$$

Thus $B=\left\{z \in \mathbb{C}^{n}:|z|<1\right\}$. We denote the open unit disk in the complex plane by $D$. Let $\operatorname{Aut}(B)$ be the group of all biholomorphic self-maps of $B$. It is well known that $\operatorname{Aut}(B)$ is generated by the unitary operators on $\mathbb{C}^{n}$ and the involutions $\varphi_{a}$ of the form

$$
\varphi_{a}(z)=\frac{a-P_{a} z-s_{a} Q_{a} z}{1-\langle z, a\rangle}
$$

where $s_{a}=\sqrt{1-|a|^{2}}, P_{a}$ is the orthogonal projection into the space spanned by $a$ and $Q_{a}=I-P_{a}$ (see, e.g., [13]).

We denote by $H(B)$ the class of all holomorphic functions on the unit ball. For $f \in H(B)$, let $\mathcal{R} f$ denote the radial derivative of $f$, that is, $\mathcal{R} f(z)=$ $\sum_{j=1}^{n} z_{j} \frac{\partial f}{\partial z_{j}}(z)$. For $f \in H(B)$, let $\nabla f$ denote the complex gradient of $f$, i.e.,

$$
\nabla f(z)=\left(\partial f / \partial z_{1}(z), \ldots, \partial f / \partial z_{n}(z)\right) .
$$

For $f \in C^{1}(B)$, let $\widetilde{\nabla} f$ denote the invariant gradient on $B$, i.e.,

$$
(\widetilde{\nabla} f)(z)=\nabla\left(f \circ \varphi_{z}\right)(0) .
$$

Received June 14, 2010; Revised August 21, 2011.

2010 Mathematics Subject Classification. Primary 32A18; Secondary 32A37.

Key words and phrases. Besov space, Bergman space, Bloch space. 
For $f \in H(B)$ and $z \in B$, set

$$
Q_{f}(z)=\sup _{w \in \mathbb{C}^{n} \backslash\{0\}} \frac{|\langle\nabla f(z), \bar{w}\rangle|}{\sqrt{\frac{n+1}{2} \frac{\left(1-|z|^{2}\right)|w|^{2}+|\langle w, z\rangle|^{2}}{\left(1-|z|^{2}\right)^{2}}}} .
$$

The Bloch space $\mathcal{B}$ is the space of all $f \in H(B)$ such that (see, e.g., [10])

$$
\|f\|_{\mathcal{B}}=\sup _{z \in B} Q_{f}(z)<\infty .
$$

Let $d v$ be the normalized Lebesgue measure of $B$. Suppose $0<p<\infty$, recall that the Bergman space $A^{p}$ consists of those $f \in H(B)$ for which

$$
\|f\|_{A^{p}}^{p}=\int_{B}|f(z)|^{p} d v(z)<\infty .
$$

For $1<p<\infty$ the Möbius invariant Besov space $B_{p}$ consists of those holomorphic functions $f$ such that

$$
\|f\|_{B_{p}}^{p}=\int_{B} Q_{f}^{p}(z) d \lambda(z)<\infty
$$

where $d \lambda(z)=\left(1-|z|^{2}\right)^{-n-1} d v(z)$ is a Möbius invariant measure, that is, for any $\psi \in \operatorname{Aut}(B)$ and $f \in L^{1}(B)$,

$$
\int_{B} f(z) d \lambda(z)=\int_{B} f \circ \psi(z) d \lambda(z) .
$$

From [1], the Besov space is nontrival if and only if $p>2 n$ when $n>1$.

It is very important to give more characterizations for a function space. Some new characterizations may be useful to study operator theory. For example, an $f \in H(B)$ is said to belong to the $\alpha$-Bloch space, denoted by $\mathcal{B}^{\alpha}(B)$, if

$$
\sup _{z \in B}\left(1-|z|^{2}\right)^{\alpha}|\nabla f(z)|<\infty .
$$

The last formula is simple. However it is difficult to study composition operators on $\alpha$-Bloch space by using this characterization. In [11], Zhang and Xu introduced the following metric (see [11] for more details)

$$
F_{z}^{\alpha}(w)=\sqrt[2]{\frac{n+1}{2}} \frac{\sqrt[2]{\lambda_{\alpha}(|z|)|w|^{2}+\left(1-\lambda_{\alpha}(|z|)\right)|\langle w, z\rangle|^{2} /|z|^{2}}}{\left(1-|z|^{2}\right)^{\alpha}} .
$$

Using this new metric, they obtained a new characterization for $\alpha$-Bloch space, i.e., they proved that $f \in \mathcal{B}^{\alpha}(B)$ if and only if

$$
\sup _{z, w \in \mathbb{C}^{n} \backslash\{0\}} \frac{|\nabla f(z) w|}{F_{z}^{\alpha}(w)}<\infty .
$$

Using (1), the boundedness and compactness of composition operators on $\alpha$ Bloch spaces have been completely characterized.

In [7], the author proved that $f \in B_{p}$ if and only if

(2) $\int_{B} \int_{B}\left(\frac{|f(z)-f(w)|}{\left|w-P_{w} z-s_{w} Q_{w} z\right|}\right)^{p}\left(1-|z|^{2}\right)^{p / 2}\left(1-|w|^{2}\right)^{p / 2} d \lambda(z) d \lambda(w)<\infty$. 
Notice that when $n=1$, we have that $P_{w}=I$ and $Q_{w}=0$, so that the denominator in (2) is exactly $|w-z|$. Therefore (2) can be seen as a generalization of the result given by Stroethoff (see [9]). In [5], we proved that $f \in B_{p}$ if and only if

$$
\int_{B} \int_{B}\left(\frac{|f(z)-f(w)|}{|1-\langle z, w\rangle|}\right)^{p}\left(1-|z|^{2}\right)^{p / 2}\left(1-|w|^{2}\right)^{p / 2} d \lambda(z) d \lambda(w)<\infty .
$$

See $[1,2,3,4,5,7,9,12,13]$ for more characterizations of the Besov space.

In this paper, we give some derivative-free characterizations for the Besov space in the unit ball of $\mathbb{C}^{n}$. Some characterizations generalized the results in the literature. Furthermore, these characterizations are new even in the unit disk.

Throughout this paper, constants are denoted by $C$, they are positive and may differ from one occurrence to the other. The notation $A \asymp B$ means that there is a positive constant $C$ such that $B / C \leq A \leq C B$.

\section{Main results and proofs}

In this section, we state our main results and proofs. We begin with the following estimate.

Lemma $1([8])$. Let $-1<t<\infty$. If $c>0$, then there is a positive constant C such that

$$
\int_{B} \frac{\left(1-|z|^{2}\right)^{t}}{|1-\langle z, w\rangle|^{n+1+t+c}} d v(z) \leq \frac{C}{\left(1-|w|^{2}\right)^{c}}
$$

for all $w \in B$.

Lemma 2 ([13]). Suppose $p>2 n$ and $f \in H(B)$. Then the following are equivalent.

(i) $f \in B_{p}$;

(ii)

(iii)

$$
\int_{B}\left(1-|w|^{2}\right)^{p}|\nabla f(w)|^{p} d \lambda(w)<\infty
$$

$$
\int_{B}|\widetilde{\nabla} f(w)|^{p} d \lambda(w)<\infty .
$$

Lemma $3([6])$. Assume that $f \in H(B), 0<p<\infty,-1<q<\infty, 0 \leq s, t<$ $\infty$ such that $p+s>n$ and $p+2 n>t$. Then for $a \in B$,

$$
\begin{aligned}
& \int_{B} \frac{|f(z)-f(0)|^{p}}{|z|^{t}}\left(1-|z|^{2}\right)^{q}\left(1-\left|\varphi_{a}(z)\right|^{2}\right)^{s} d v(z) \\
\leq & C \int_{B}|\mathcal{R} f|^{p}\left(1-|z|^{2}\right)^{p+q}\left(1-\left|\varphi_{a}(z)\right|^{2}\right)^{s} d v(z) \\
\leq & C \int_{B}|\widetilde{\nabla} f|^{p}\left(1-|z|^{2}\right)^{q}\left(1-\left|\varphi_{a}(z)\right|^{2}\right)^{s} d v(z) .
\end{aligned}
$$


Now we are in a position to state and prove the main results of this paper.

Theorem 1. Assume that $f \in H(B), p>2 n, n+1 \leq c, t<\infty$. Then $f \in B_{p}$ if and only if

$$
\int_{B} \int_{B} \frac{|f(z)-f(w)|^{p}}{|1-\langle z, w\rangle|^{t+c}}\left(1-|z|^{2}\right)^{t}\left(1-|w|^{2}\right)^{c} d \lambda(z) d \lambda(w)<\infty .
$$

Proof. Suppose that (5) holds. For a fixed $r \in(0,1)$, let $E(a, r)=\{z \in B$ : $\left.\left|\varphi_{a}(z)\right|<r\right\}$. Set $|E(a, r)|=v(E(a, r))$. From [7] or [13] we see that

$$
\left(1-|a|^{2}\right)^{n+1} \asymp\left(1-|z|^{2}\right)^{n+1} \asymp|1-\langle a, z\rangle|^{n+1} \asymp|E(a, r)|
$$

when $z \in E(a, r)$. It is easy to see (using Cauchy's estimate for example) that there exists a positive constant $C$ such that

$$
|\nabla f(0)|^{p} \leq C \int_{E(0, r)}|f(w)-f(0)|^{p} d v(w)
$$

for all $f \in H(B)$, where $r$ is any fixed positive radius. Replacing $f$ by $f \circ \varphi_{z}$ and making a change of variable, we get

$$
|\widetilde{\nabla} f(z)|^{p} \leq C \int_{E(z, r)}|f(z)-f(w)|^{p} \frac{\left(1-|z|^{2}\right)^{n+1}}{|1-\langle z, w\rangle|^{2(n+1)}} d v(w)
$$

for all $f \in H(B)$ and $z \in B$. Combining the fact (6) with (7) we see that there is another positive constant $C>0$ such that

$$
\left(1-|z|^{2}\right)^{p}|\nabla f(z)|^{p} \leq C \int_{E(z, r)}|f(z)-f(w)|^{p} d \lambda(w)
$$

for all $f \in H(B)$ and $z \in B$. Then by (6) we have

$$
\left(1-|w|^{2}\right)^{p}|\nabla f(w)|^{p} \leq C \int_{E(w, r)} \frac{|f(z)-f(w)|^{p}\left(1-|z|^{2}\right)^{t-(n+1)}\left(1-|w|^{2}\right)^{c}}{|1-\langle z, w\rangle|^{t+c}} d v(z) .
$$

Hence

$$
\begin{aligned}
& \int_{B}\left(1-|w|^{2}\right)^{p}|\nabla f(w)|^{p} d \lambda(w) \\
\leq & C \int_{B} \int_{E(w, r)}|f(z)-f(w)|^{p} \frac{\left(1-|z|^{2}\right)^{t}\left(1-|w|^{2}\right)^{c}}{|1-\langle z, w\rangle|^{t+c}} d \lambda(z) d \lambda(w) \\
\leq & C \int_{B} \int_{B}|f(z)-f(w)|^{p} \frac{\left(1-|z|^{2}\right)^{t}\left(1-|w|^{2}\right)^{c}}{|1-\langle z, w\rangle|^{t+c}} d \lambda(z) d \lambda(w)<\infty .
\end{aligned}
$$

It follows from Lemma 2 that $f \in B_{p}$.

Conversely, suppose that $f \in B_{p}$. From Theorem 3 of [7],

$$
\begin{aligned}
& \int_{B} \int_{B} \frac{|f(z)-f(w)|^{p}}{|1-\langle z, w\rangle|^{t+c}}\left(1-|z|^{2}\right)^{t}\left(1-|w|^{2}\right)^{c} d \lambda(z) d \lambda(w) \\
= & \int_{B} \int_{B} \frac{|f(z)-f(w)|^{p}}{|1-\langle z, w\rangle|^{2(n+1)}} \frac{\left(1-|z|^{2}\right)^{t-(n+1)}\left(1-|w|^{2}\right)^{c-(n+1)}}{|1-\langle z, w\rangle|^{t+c-2(n+1)}} d v(z) d v(w)
\end{aligned}
$$




$$
\leq C \int_{B} \int_{B} \frac{|f(z)-f(w)|^{p}}{|1-\langle z, w\rangle|^{2(n+1)}} d v(z) d v(w)<\infty .
$$

The proof is completed.

Theorem 2. Assume that $f \in H(B), n+1 \leq c, t<\infty$ and $p>\max \{2 n, t+$ $c-2 n\}$. Then $f \in B_{p}$ if and only if

(8) $I=\int_{B} \int_{B} \frac{|f(z)-f(w)|^{p}}{\left|w-P_{w} z-s_{w} Q_{w} z\right|^{t+c}}\left(1-|z|^{2}\right)^{t}\left(1-|w|^{2}\right)^{c} d \lambda(z) d \lambda(w)<\infty$.

Proof. Sufficiency. Since

$$
\frac{1}{|1-\langle z, a\rangle|} \leq \frac{1}{\left|a-P_{a} z-s_{a} Q_{a} z\right|}, z, a \in B,
$$

the result follows from the above inequality and Theorem 1 .

Necessity. Making the change of variables $w \mapsto \varphi_{z}(w)$ and using the equality

$$
1-\left\langle\varphi_{z}(w), z\right\rangle=\frac{1-|z|^{2}}{1-\langle w, z\rangle}
$$

we obtain

$$
\begin{aligned}
& I=\int_{B} \int_{B} \frac{|f(z)-f(w)|^{p}}{\left|w-P_{w} z-s_{w} Q_{w} z\right|^{t+c}}\left(1-|z|^{2}\right)^{t}\left(1-|w|^{2}\right)^{c} d \lambda(z) d \lambda(w) \\
& =\int_{B} \int_{B} \frac{|f(z)-f(w)|^{p}}{\left|\varphi_{z}(w)\right|^{t+c}|1-\langle z, w\rangle|^{t+c}}\left(1-|z|^{2}\right)^{t}\left(1-|w|^{2}\right)^{c} d \lambda(z) d \lambda(w) \\
& =\int_{B}\left(1-|z|^{2}\right)^{t} d \lambda(z) \int_{B} \frac{\left|f \circ \varphi_{z}(w)-f \circ \varphi_{z}(0)\right|^{p}}{|w|^{t+c}\left|1-\left\langle z, \varphi_{z}(w)\right\rangle\right|^{t+c}}\left(1-\left|\varphi_{z}(w)\right|^{2}\right)^{c} d \lambda(w) \\
& \leq \int_{B} d \lambda(z) \int_{B} \frac{\left|f \circ \varphi_{z}(w)-f \circ \varphi_{z}(0)\right|^{p}\left(1-|w|^{2}\right)^{c}}{|w|^{t+c}|1-\langle z, w\rangle|^{c-t}} d \lambda(w) .
\end{aligned}
$$

Using the assumed condition $p>t+c-2 n$, it is elementary to show that there exists a positive constant $C$ (independent of $f$ ) such that

$$
I \leq C \int_{B} d \lambda(z) \int_{B} \frac{\left|f \circ \varphi_{z}(w)-f \circ \varphi_{z}(0)\right|^{p}\left(1-|w|^{2}\right)^{c}}{|1-\langle z, w\rangle|^{c-t}} d \lambda(w) .
$$

Making the change of variables $w \mapsto \varphi_{z}(w)$ and using the equality (10) again, we obtain

$$
I \leq C \int_{B} \int_{B} \frac{|f(z)-f(w)|^{p}}{|1-\langle z, w\rangle|^{t+c}}\left(1-|z|^{2}\right)^{t}\left(1-|w|^{2}\right)^{c} d \lambda(z) d \lambda(w) .
$$

From Theorem 1 we get that $I<\infty$, as desired. The proof is completed.

Remark 1. Taking $t=c=n+1$ in Theorem 1 , we get Theorem 3 of [7]. Hence Theorem 1 can be regarded as an extension of the result in [7]. In particular, taking $t=c=p / 2$ in Theorems 1 and 2, we get (3) and (2) respectively.

From Theorems 1 and 2, we get the following characterizations of the Besov space in the unit disk. 
Corollary 1. Assume that $f \in H(D), 2 \leq c, t<\infty$ and $p>2$. Then $f \in B_{p}(D)$ if and only if

$$
\int_{D} \int_{D} \frac{|f(z)-f(w)|^{p}}{|1-\bar{w} z|^{t+c}}\left(1-|z|^{2}\right)^{t}\left(1-|w|^{2}\right)^{c} d \tau(z) d \tau(w)<\infty,
$$

where $d \tau=\left(1-|z|^{2}\right)^{-2} d A(z)$ is the Möbius invariant measure and $d A(z)$ is the Lebesgue measure on the unit disk.

Corollary 2. Assume that $f \in H(D), 2 \leq c, t<\infty$ and $p>\max \{2, t+c-2\}$. Then $f \in B_{p}(D)$ if and only if

$$
\int_{D} \int_{D} \frac{|f(z)-f(w)|^{p}}{|w-z|^{t+c}}\left(1-|z|^{2}\right)^{t}\left(1-|w|^{2}\right)^{c} d \tau(z) d \tau(w)<\infty .
$$

Theorem 3. Assume that $f \in H(B), p>2 n$. Then $f \in B_{p}$ if and only if (11)

$$
\int_{B} \int_{B} \int_{B} \frac{|f(z)-f(w)|^{p}}{|1-\langle z, w\rangle|^{2(n+1)}}\left(1-\left|\varphi_{a}(z)\right|^{2}\right)^{\frac{n+1}{2}}\left(1-\left|\varphi_{a}(w)\right|^{2}\right)^{\frac{n+1}{2}} d v(z) d v(w) d \lambda(a)<\infty .
$$

Proof. Suppose that (11) holds. For a fixed $r \in(0,1)$, when $z \in E(w, r)$, it holds

$$
|1-\langle z, a\rangle| \asymp|1-\langle w, a\rangle|,
$$

for any $a \in B$ (see [13]). Using (12), from the proof of Theorem 1 we have

$$
\begin{aligned}
& \left(1-|w|^{2}\right)^{p}|\nabla f(w)|^{p}\left(1-\left|\varphi_{a}(w)\right|^{2}\right)^{n+1} \\
\leq & C \int_{E(w, r)} \frac{|f(z)-f(w)|^{p}}{|1-\langle z, w\rangle|^{2(n+1)}}\left(1-|w|^{2}\right)^{n+1}\left(1-\left|\varphi_{a}(w)\right|^{2}\right)^{\frac{n+1}{2}}\left(1-\left|\varphi_{a}(z)\right|^{2}\right)^{\frac{n+1}{2}} d v(z) .
\end{aligned}
$$

Hence

$$
\begin{aligned}
& \int_{B}\left(1-|w|^{2}\right)^{p}|\nabla f(w)|^{p}\left(1-\left|\varphi_{a}(w)\right|^{2}\right)^{n+1} d \lambda(w) \\
\leq & C \int_{B} \int_{B} \frac{|f(z)-f(w)|^{p}}{|1-\langle z, w\rangle|^{2(n+1)}}\left(1-\left|\varphi_{a}(z)\right|^{2}\right)^{\frac{n+1}{2}}\left(1-\left|\varphi_{a}(w)\right|^{2}\right)^{\frac{n+1}{2}} d v(z) d v(w) .
\end{aligned}
$$

Therefore

$$
\begin{aligned}
& \int_{B}\left(1-|w|^{2}\right)^{p}|\nabla f(w)|^{p} d \lambda(w) \\
&= \int_{B}\left(1-|w|^{2}\right)^{p}|\nabla f(w)|^{p} d \lambda(w) \int_{B}\left(1-\left|\varphi_{w}(a)\right|^{2}\right)^{n+1} d \lambda(a) \\
&= \int_{B} \int_{B}\left(1-|w|^{2}\right)^{p}|\nabla f(w)|^{p}\left(1-\left|\varphi_{a}(w)\right|^{2}\right)^{n+1} d \lambda(w) d \lambda(a) \\
& \leq C \int_{B} \int_{B} \int_{B} \frac{|f(z)-f(w)|^{p}}{|1-\langle z, w\rangle|^{2(n+1)}}\left(1-\left|\varphi_{a}(z)\right|^{2}\right)^{\frac{n+1}{2}}\left(1-\left|\varphi_{a}(w)\right|^{2}\right)^{\frac{n+1}{2}} d v(z) d v(w) d \lambda(a) \\
&< \infty \\
& \text { i.e., } f \in B_{p}, \text { as desired. }
\end{aligned}
$$


Conversely, suppose that $f \in B_{p}$. We first claim that for any $g \in A^{p}$,

$$
\begin{aligned}
Y & =\int_{B} \int_{B} \frac{|g(z)-g(w)|^{p}}{|1-\langle z, w\rangle|^{2(n+1)}}\left(1-|z|^{2}\right)^{\frac{n+1}{2}}\left(1-|w|^{2}\right)^{\frac{n+1}{2}} d v(z) d v(w) \\
& \leq C \int_{B}|g(z)|^{p} d v(z) \asymp \int_{B}|\widetilde{\nabla} g(z)|^{p} d v(z)<\infty .
\end{aligned}
$$

In fact, using Lemma 1 we obtain

$$
\begin{aligned}
Y \leq & C \int_{B} \int_{B} \frac{|g(z)|^{p}}{|1-\langle z, w\rangle|^{2(n+1)}}\left(1-|z|^{2}\right)^{\frac{n+1}{2}}\left(1-|w|^{2}\right)^{\frac{n+1}{2}} d v(z) d v(w) \\
& +C \int_{B} \int_{B} \frac{|g(w)|^{p}}{|1-\langle z, w\rangle|^{2(n+1)}}\left(1-|z|^{2}\right)^{\frac{n+1}{2}}\left(1-|w|^{2}\right)^{\frac{n+1}{2}} d v(z) d v(w) \\
\leq & C \int_{B}|g(z)|^{p}\left(1-|z|^{2}\right)^{\frac{n+1}{2}} d v(z) \int_{B} \frac{\left(1-|w|^{2}\right)^{\frac{n+1}{2}}}{|1-\langle z, w\rangle|^{2(n+1)}} d v(w) \\
& +C \int_{B}|g(w)|^{p}\left(1-|w|^{2}\right)^{\frac{n+1}{2}} d v(w) \int_{B} \frac{\left(1-|z|^{2}\right)^{\frac{n+1}{2}}}{|1-\langle z, w\rangle|^{2(n+1)}} d v(z) \\
\leq & C \int_{B}|g(z)|^{p} d v(z)+C \int_{B}|g(w)|^{p} d v(w) \leq C \int_{B}|g(z)|^{p} d v(z) .
\end{aligned}
$$

For $f \in B_{p} \subset \mathcal{B}$ and $a \in B$, we have that $f \circ \varphi_{a}-f(a) \in A^{p}$ (see, e.g., [13]). From (13) we have

$$
\begin{aligned}
& \int_{B} \int_{B} \frac{\left|f \circ \varphi_{a}(z)-f \circ \varphi_{a}(w)\right|^{p}}{|1-\langle z, w\rangle|^{2(n+1)}}\left(1-|z|^{2}\right)^{\frac{n+1}{2}}\left(1-|w|^{2}\right)^{\frac{n+1}{2}} d v(z) d v(w) \\
\leq & C \int_{B}\left|\widetilde{\nabla} f\left(\varphi_{a}(z)\right)\right|^{p} d v(z)=C \int_{B}|\widetilde{\nabla} f(z)|^{p}\left(1-\left|\varphi_{a}(z)\right|^{2}\right)^{n+1} d \lambda(z) .
\end{aligned}
$$

Making the change of variables $z \mapsto \varphi_{a}(z), w \mapsto \varphi_{a}(w)$ and using the following equality (see [13])

$$
\frac{\left(1-\left|\varphi_{a}(z)\right|^{2}\right)\left(1-\left|\varphi_{a}(w)\right|^{2}\right)}{\left|1-\left\langle\varphi_{a}(z), \varphi_{a}(w)\right\rangle\right|^{2}}=1-\left|\varphi_{w}(z)\right|^{2}
$$

we obtain

$$
\begin{aligned}
& \int_{B} \int_{B} \int_{B} \frac{|f(z)-f(w)|^{p}}{|1-\langle z, w\rangle|^{2(n+1)}}\left(1-\left|\varphi_{a}(z)\right|^{2}\right)^{\frac{n+1}{2}}\left(1-\left|\varphi_{a}(w)\right|^{2}\right)^{\frac{n+1}{2}} d v(z) d v(w) d \lambda(a) \\
\leq & C \int_{B} \int_{B}|\widetilde{\nabla} f(z)|^{p}\left(1-\left|\varphi_{a}(z)\right|^{2}\right)^{n+1} d \lambda(z) d \lambda(a) \\
= & C \int_{B}|\widetilde{\nabla} f(z)|^{p} d \lambda(z)<\infty .
\end{aligned}
$$

The proof is finished. 
Theorem 4. Assume that $f \in H(B), p>2 n$. Then $f \in B_{p}$ if and only if (14) $\int_{B} \int_{B} \int_{B} \frac{|f(z)-f(w)|^{p}\left(1-\left|\varphi_{a}(z)\right|^{2}\right)^{\frac{n+1}{2}}\left(1-\left|\varphi_{a}(w)\right|^{2}\right)^{\frac{n+1}{2}}}{\left|w-P_{w} z-s_{w} Q_{w} z\right|^{2(n+1)}} d v(z) d v(w) d \lambda(a)<\infty$.

Proof. Sufficiency. The result follows from Theorem 3 and (9).

Necessity. Suppose that $f \in B_{p}$. For $a \in B$, by Lemma 3 we have

$$
\begin{aligned}
& \int_{B} \int_{B} \frac{|f(z)-f(w)|^{p}}{\left|w-P_{w} z-s_{w} Q_{w} z\right|^{2(n+1)}}\left(1-\left|\varphi_{a}(z)\right|^{2}\right)^{\frac{n+1}{2}}\left(1-\left|\varphi_{a}(w)\right|^{2}\right)^{\frac{n+1}{2}} d v(z) d v(w) \\
= & \int_{B} \int_{B} \frac{|f(z)-f(w)|^{p}}{\left|\varphi_{w}(z)\right|^{2(n+1)}|1-\langle w, z\rangle|^{2(n+1)}}\left(1-\left|\varphi_{a}(z)\right|^{2}\right)^{\frac{n+1}{2}}\left(1-\left|\varphi_{a}(w)\right|^{2}\right)^{\frac{n+1}{2}} d v(z) d v(w) \\
= & \int_{B} \int_{B} \frac{\left|f \circ \varphi_{w}(u)-f \circ \varphi_{w}(0)\right|^{p}}{|u|^{2(n+1)}}\left(1-\left|\varphi_{a}\left(\varphi_{w}(u)\right)\right|^{2}\right)^{\frac{n+1}{2}} d v(u)\left(1-\left|\varphi_{a}(w)\right|^{2}\right)^{\frac{n+1}{2}} d \lambda(w) \\
\leq & C \int_{B} \int_{B}\left|\widetilde{\nabla} f \circ \varphi_{w}(u)\right|^{p}\left(1-\left|\varphi_{a}\left(\varphi_{w}(u)\right)\right|^{2}\right)^{\frac{n+1}{2}} d v(u)\left(1-\left|\varphi_{a}(w)\right|^{2}\right)^{\frac{n+1}{2}} d \lambda(w) \\
\leq & C \int_{B} \int_{B}|\widetilde{\nabla} f(z)|^{p}\left(1-\left|\varphi_{w}(z)\right|^{2}\right)^{n+1}\left(1-\left|\varphi_{a}(z)\right|^{2}\right)^{\frac{n+1}{2}} d \lambda(z)\left(1-\left|\varphi_{a}(w)\right|^{2}\right)^{\frac{n+1}{2}} d \lambda(w) \\
\leq & C \int_{B}|\widetilde{\nabla} f(z)|^{p}\left(1-\left|\varphi_{a}(z)\right|^{2}\right)^{n+1} d \lambda(z) \times J .
\end{aligned}
$$

Here

$$
J=\sup _{a, z \in B} \int_{B} \frac{1}{\left(1-\left|\varphi_{a}(z)\right|^{2}\right)^{\frac{n+1}{2}}}\left(1-\left|\varphi_{w}(z)\right|^{2}\right)^{n+1}\left(1-\left|\varphi_{a}(w)\right|^{2}\right)^{\frac{n+1}{2}} d \lambda(w) .
$$

Making the change of variables $w \mapsto \varphi_{z}(u)$ and using the fact that $\left|\varphi_{z}(w)\right|=$ $\left|\varphi_{w}(z)\right|$ we have

$$
J=\sup _{a, z \in B} \int_{B} \frac{1}{\left(1-\left|\varphi_{z}(a)\right|^{2}\right)^{\frac{n+1}{2}}}\left(1-|u|^{2}\right)^{n+1}\left(1-\left|\varphi_{a}\left(\varphi_{z}(u)\right)\right|^{2}\right)^{\frac{n+1}{2}} d \lambda(u) .
$$

From the exercises 1.24 of [13] we see that $\left|\varphi_{a}\left(\varphi_{z}(u)\right)\right|=\left|\varphi_{\varphi_{z}(a)}(u)\right|$. It follows from Theorem 1.12 of [13] that

$$
\begin{aligned}
J & =\sup _{a, z \in B} \int_{B} \frac{1}{\left(1-\left|\varphi_{z}(a)\right|^{2}\right)^{\frac{n+1}{2}}}\left(1-\left|\varphi_{\varphi_{z}(a)}(u)\right|^{2}\right)^{\frac{n+1}{2}} d v(u) \\
& =\sup _{a, z \in B} \int_{B} \frac{\left(1-|u|^{2}\right)^{\frac{n+1}{2}}}{\left|1-\left\langle u, \varphi_{z}(a)\right\rangle\right|^{n+1}} d v(u) \\
& =\sup _{w \in B} \int_{B} \frac{\left(1-|u|^{2}\right)^{\frac{n+1}{2}}}{|1-\langle u, w\rangle|^{n+1}} d v(u)<\infty .
\end{aligned}
$$

Combining (15) with (16), we get

$$
\int_{B} \int_{B} \int_{B} \frac{|f(z)-f(w)|^{p}\left(1-\left|\varphi_{a}(z)\right|^{2}\right)^{\frac{n+1}{2}}\left(1-\left|\varphi_{a}(w)\right|^{2}\right)^{\frac{n+1}{2}}}{\left|w-P_{w} z-s_{w} Q_{w} z\right|^{2(n+1)}} d v(z) d v(w) d \lambda(a)
$$




$$
\begin{aligned}
& \leq C \int_{B} \int_{B}|\widetilde{\nabla} f(z)|^{p}\left(1-\left|\varphi_{a}(z)\right|^{2}\right)^{n+1} d \lambda(z) d \lambda(a) \\
& \leq C \int_{B}|\widetilde{\nabla} f(z)|^{p} d \lambda(z)<\infty .
\end{aligned}
$$

The proof is completed.

Similarly, we have the following characterizations of the Besov space in the unit disk.

Corollary 3. Assume that $f \in H(D)$ and $p>2$. Then the following are equivalent.

(i) $f \in B_{p}(D)$;

(ii)

$$
\int_{D} \int_{D} \int_{D} \frac{|f(z)-f(w)|^{p}}{|1-\bar{w} z|^{4}}\left(1-\left|\varphi_{a}(z)\right|^{2}\right)\left(1-\left|\varphi_{a}(w)\right|^{2}\right) d A(z) d A(w) d \tau(a)<\infty ;
$$

$$
\int_{D} \int_{D} \int_{D} \frac{|f(z)-f(w)|^{p}}{|z-w|^{4}}\left(1-\left|\varphi_{a}(z)\right|^{2}\right)\left(1-\left|\varphi_{a}(w)\right|^{2}\right) d A(z) d A(w) d \tau(a)<\infty .
$$

Acknowledgments. The author thanks the referee for several comments and the correction numerous mistakes for the first version of the paper. The project supported by Guangdong Natural Science Foundation(No.10451401501004305), National Natural Science Foundation of China(No.11001107) and Foundation for Distinguished Young Talents in Higher Education of Guangdong, China (LYM11117).

\section{References}

[1] K. Hahn and E. Youssfi, Möbius invariant Besov p-spaces and Hankel operators in the Bergman space on the ball in $\mathbb{C}^{n}$, Complex Variables Theory Appl. 17 (1991), no. 1-2, 89-104.

[2] H. T. Kaptanoglu, Bergman projections on Besov spaces on balls, Illinois J. Math. 49 (2005), no. 2, 385-403.

[3] _ Carleson measures for Besov spaces on the ball with applications, J. Funct. Anal. 250 (2007), no. 2, 483-520.

[4] E. G. Kwon, A characterization of Bloch space and Besov space, J. Math. Anal. Appl. 324 (2006), no. 2, 1429-1437.

[5] S. Li and S. Stević, Some characterizations of the Besov space and the $\alpha$-Bloch space, J. Math. Anal. Appl. 346 (2008), no. 1, 262-273.

[6] S. Li and H. Wulan, Some new characterizations of Bloch spaces, Taiwanese J. Math. 14 (2010), no. 6, 2245-2259.

[7] M. Nowak, Bloch and Möbius invariant Besov spaces on the unit ball of $\mathbb{C}^{n}$, Complex Variables Theory Appl. 44 (2001), no. 1, 1-12.

[8] W. Rudin, Function Theory in the Unit Ball of $\mathbb{C}^{n}$, Springer-Verlag, New York, 1980.

[9] K. Stroethoff, The Bloch space and Besov spaces of analytic functions, Bull. Austral. Math. Soc. 54 (1996), no. 2, 211-219.

[10] R. M. Timoney, Bloch functions in several complex variables II, J. Reine Angew. Math. 319 (1980), 1-22. 
[11] M. Zhang and W. Xu, Composition operators on $\alpha$-Bloch spaces of the unit ball, Acta Math. Sin. (Engl. Ser.) 23 (2007), no. 11, 1991-2002.

[12] K. Zhu, Analytic Besov space, J. Math. Anal. Appl. 157 (1991), no. 2, 318-336.

[13] Spaces of Holomorphic Functions in the Unit Ball, Springer-Verlag, New York, 2005.

Department of Mathematics

JIAYING UNIVERSITY

514015, Meizhou, GuangDong, P. R. China

E-mail address: jyulsx@163.com, lsx@mail.zjxu.edu.cn 